\title{
Corrigendum: Genome-wide association study identifies variants at $16 p 13$ associated with survival in multiple myeloma patients
}

Elad Ziv, Eric Dean, Donglei Hu, Alessandro Martino, Daniel Serie, Karen Curtin, Daniele Campa, Blake Aftab, Paige Bracci, Gabriele Buda, Yi Zhao, Jennifer Caswell-Jin, Robert Diasio, Charles Dumontet, Marek Dudziński, Laura Fejerman, Alexandra Greenberg, Scott Huntsman, Krzysztof Jamroziak, Artur Jurczyszyn, Shaji Kumar, Djordje Atanackovic, Martha Glenn, Lisa A. Cannon-Albright, Brandt Jones, Adam Lee, Herlander Marques, Thomas Martin, Joaquin Martinez-Lopez, Vincent Rajkumar, Juan Sainz, Annette Juul Vangsted, Marzena Watek, Jeffrey Wolf, Susan Slager, Nicola J. Camp, Federico Canzian \& Celine Vachon

Nature Communications 6:7539 doi: 10.1038/ncomms8539 (2015); Published 22 Jul 2015; Updated 9 Dec 2015

In this Article, members of the UCSF cohort who had been alive for longer than two years were inadvertently included in the data presented in Table 3. USCF/old treatments should have 109 patients with a hazard ratio of 3.35 and a $P$ value of 0.00028 instead of the 124 patients with a hazard ratio of 3.37 and a $P$ value of 0.00026 . The USCF/new patients should have 187 patients with a hazard ratio of 3.57 and a $P$ value of 0.0007 instead of the 208 patients with a hazard ratio of 3.62 and a $P$ value of 0.0006 . Finally, in the table legend, the first line should read 'All models are adjusted for age, gender and principal components 1-3'. The exclusion of these individuals does not change the conclusions of the study. The correct version of Table 3 appears below.

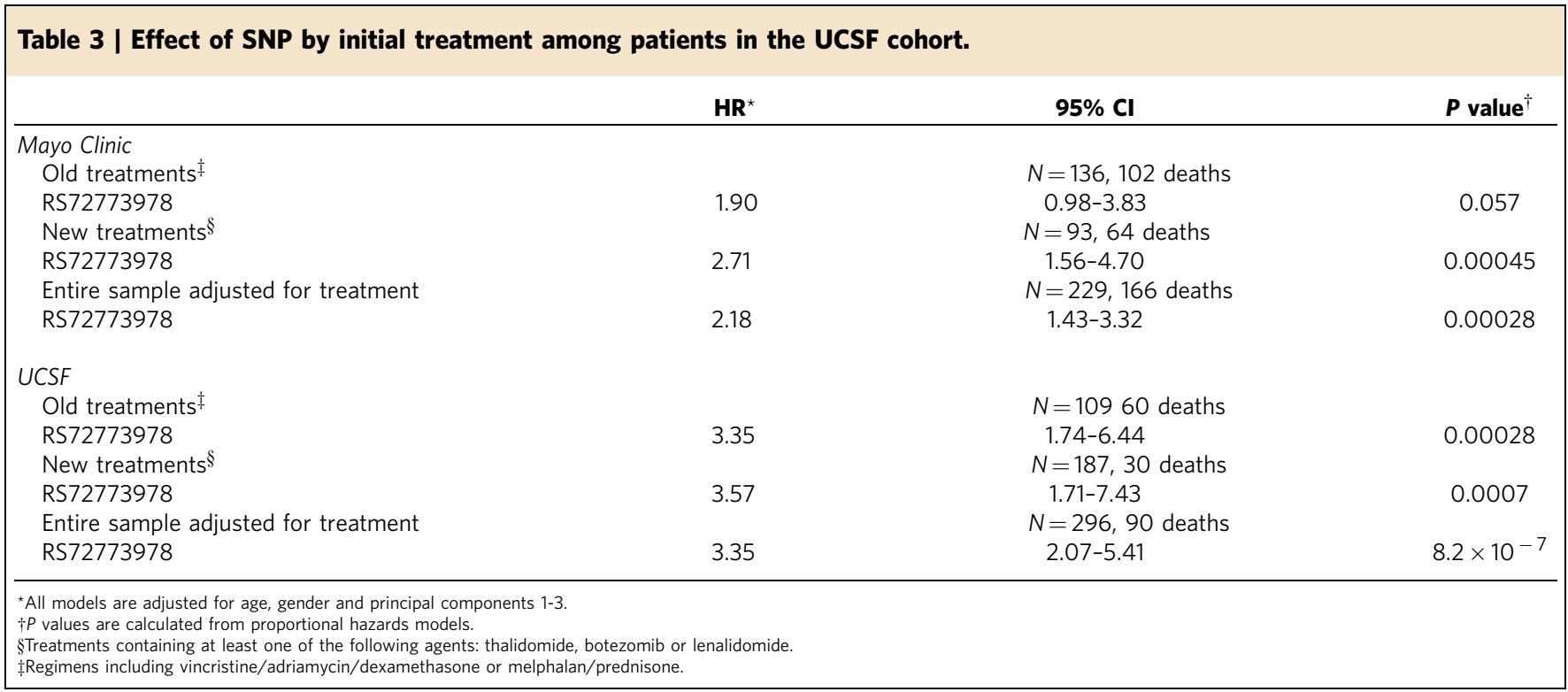

